\title{
The Multiple Ingredients Effect: The More Diverse (Un)Healthy Ingredients, the More (Un)Healthy the Food
}

This article was published in the following Dove Press journal: Psychology Research and Behavior Management

\section{Yunjoo Jeong \\ Sanyoung Hwang \\ Mijin Kwon}

Business, Korea University, Seoul, Republic of Korea
Correspondence: Sanyoung Hwang Business, Korea University, I45, Anam-ro, Seongbuk-gu, Seoul 0284I, Republic of Korea

Email sanyoung120@korea.ac.kr
Background: Although previous research has demonstrated that ingredient information plays a significant role in consumers' healthier food choices, none of the research has investigated how the number of ingredients (ie, single vs multiple) influences consumer perceptions of food healthiness. However, this research argues that consumers have lay belief that the greater the number of (un)healthy ingredients in food, the more (un)healthy the food is perceived and they rely on the lay belief when judging food healthiness. Thus, this paper proposes and examines the multiple ingredients effect that people make inferences about food (un)healthiness based on the diversity of (un)healthy ingredients.

Methods and Results: Four studies test our hypotheses using ANOVAs and regression analyses. Study 1 examines that people indicate a higher perception of food unhealthiness when the number of unhealthy ingredients is presented as multiple rather than single. Study 2 replicates the multiple ingredients effect in the healthy food domain and eliminates an alternative explanation based on the subadditivity effect. Study 3 also finds that the difference in food healthiness perception between people who have high and low health consciousness is driven by the salience of each ingredient. Finally, Study 4 identifies the evaluability of the nutritional value as a boundary condition for our effect in an AI-based self-service context.

Conclusion: The current research demonstrates the multiple ingredients effect that people perceive higher food (un)healthiness when the number of (un)healthy ingredients is greater although nutritional information is identically presented. Moreover, this effect is moderated by the extent to which people are conscious of health-related issues. This finding is because ingredient information is highly accessible and salient for health-conscious people .

Keywords: food healthiness perception, heuristic processing, lay belief, artificial intelligence

\section{Introduction}

Imagine a person is about to order pizza in a restaurant. In the menu, there are two types of pizza with variations of meat toppings, providing saturated fat information on each topping: Pizza A is topped with sausage $(5 \mathrm{~g})$, bacon $(5 \mathrm{~g})$, and ham $(5 \mathrm{~g})$ while Pizza B is topped with only sausage (15g). Given that the total amount of saturated fat is equal between pizza $\mathrm{A}$ and $\mathrm{B}$, which one seems to be unhealthier?

As a result of government policies and actions, consumers nowadays can easily find detailed nutrition information on food packages, including ingredients, item size, calories, nutrition label, and so on. Some studies have shown that the presence 
of nutritional content has a positive effect on the quality of consumer food consumption. ${ }^{1-3}$ However, consumers often find themselves making biased food decisions because of the tendency to rely on their existing cognitive structures, such as lay beliefs, which causes them to fail to engage in systematic processing to understand presented information evenly. ${ }^{4-7}$

Ingredient information plays a significant role in consumers' healthier food choices. According to the consumer survey conducted by ingredients manufacturer Beneo, more than half of the consumers (51\%) indicated ingredient lists as an important factor in making healthier food decisions. ${ }^{8}$ Marketers often make health claims that promote particular ingredients to emphasize the healthiness of their food products (eg, "Nature Valley Crunchy with Pumpkin \& Poppy Seeds"). Despite the role of the ingredient as an important food purchasing motivator, none of the research has investigated how the number of ingredients (ie, single vs multiple) influences consumer perceptions of food healthiness. Thus, the current research proposes that people rely on the lay belief that "the greater the number of (un)healthy ingredients, the more (un) healthy the food." Considering that consumers are unlikely to process every information on food products systematically, they tend to engage in heuristic processing with existing lay theories. ${ }^{9,10}$ Therefore, this research suggests the multiple ingredients effect, which posits that people have a tendency to infer food healthiness from the number of (un)healthy ingredients in food. Additionally, it is predicted that the multiple ingredients effect will be more pronounced for highly health-conscious people as compared to less health-conscious people.

Perceived healthiness of foods has been one of the important determinants of consumer food consumption. ${ }^{11-14}$ For instance, Chandon and Wansink ${ }^{12}$ showed that perceiving the restaurant as offering healthy foods affects the choice of side dishes. In this sense, an examination of whether a food product is perceived to be healthy can be a useful means of anticipating consumers' actual food choice and consumption. Thus, it is expected that our research provides meaningful implications for marketers and policymakers in the field of consumer welfare. Additionally, reflecting on the widespread deployment of "untact" (ie, undo contact) self-service devices, ${ }^{15}$ the study explores consumers' food decision-making in an AI-based self-service context. The present research suggests that providing reference information via AI-enabled chatbot allows consumers to make unbiased food decisions, thereby reducing the difficulty of evaluating unfamiliar nutrition information of food products. Hence, the current research offers practical guidance for consumer welfare.

\section{Literature Review and Hypothesis Development Multiple Ingredients Effect: Diversity of Ingredients as a Heuristic Cue}

Consumers are often biased by a variety of cues when making food-related decisions. For example, serving sizes, ${ }^{16}$ brand names, ${ }^{12}$ and nutrition information on package $^{17}$ act as a bias that affects healthiness perception and purchase intention on food. Food information on the presence of particular ingredients (eg, natural honey and almonds in Greek yogurt) itself can be also useful yet biasing cues for assessing the healthiness of food products. Indeed, cereals containing "fruit sugar" are perceived to be healthier than cereals containing "sugar" because the "fruit" generally symbolizes healthiness and is thus considered as a healthiness cue. ${ }^{18}$ Adding on the relationship between the presence of ingredients and food perception, the current study suggests that people infer food (un) healthiness from the number of (un)healthy ingredients in food. Thus, this research posits that not only the presence of the ingredient itself but also the sheer number of ingredients can affect the perception of food healthiness.

Previous studies show that the number of distinct information acts as a diagnostic cue for judgment. ${ }^{19-22}$ For example, Petty and Cacioppo ${ }^{19}$ find that increasing the mere number of arguments has a positive influence on message persuasion because people employ the simple intuition "the more the better." This intuition can be applied when evaluating supplement efficacy. Homer and Mukherjee ${ }^{20}$ demonstrate that people perceive the multiple supplements are more effective than a single supplement. In a similar vein, Berger, Draganska, and Simonson ${ }^{21}$ suggest that the diversity serves as a cue for evaluating brand quality and they find that increasing the number of options a brand offers leads consumers to perceive the brand as more expertise or having a higher quality. Oppewal and Koelemeijer ${ }^{22}$ show similar findings of the positive association between the number of option and evaluation. This is because when the number of components increases, people pay more attention to the components, ${ }^{23,24}$ leading to a greater reliance on them. In line with these findings, it is suggested that people would 
apply "the more the better" intuition when assessing food healthiness, which leads to our proposition that people have a lay belief that "the greater the number of (un) healthy ingredients in food, the more (un)healthy the food." The current research directly examines the lay belief about the association between the number of (un) healthy ingredients and food healthiness by conducting pretests. The results of the pretests (details will be discussed later) show that a majority of respondents (83.6\%) believed that the diversity of (un)healthy ingredients influences food healthiness.

Considering that we have more than 1000 meals a year, putting effort into every food decision is not easy, making it highly likely that people engage in heuristic processing when making food-related decisions. ${ }^{25}$ This heuristic information processing leads them to rely on existing cognitive structures, such as lay beliefs, ${ }^{9,10}$ commonly used to understand the world. ${ }^{26}$ Thus, this research argues that people will judge food healthiness by relying on the intuitive association of the number of (un)healthy ingredients and food (un)healthiness. Specifically, the source of the proposed lay theory is consistent with the notion of availability heuristics that frequent exposure to certain information leads to the high accessibility of information. ${ }^{27}$ In the case of judging whether the food is healthy, judgments are affected by frequently encountered ingredient information. Therefore, the present research posits that people are likely to employ the learned association between the number of (un)healthy ingredients and food healthiness as a heuristic cue when assessing food healthiness.

The reliance on lay beliefs is enhanced when people lack the prior knowledge required for systematic processing of unfamiliar information. ${ }^{4,9,10}$ Prior research has shown that people are more likely to employ the sheer number of information to make judgments when they do not have the ability to understand given information. ${ }^{19-22}$ Returning to our opening anecdote on the person ordering pizza, which nutritional information between ingredients and saturated fat will be used as a basis for assessing the healthiness of pizza? Although the nutritional value (eg, fat content) of food products is an accurate and objective indicator of food healthiness, it is predicted that people will ignore this core information and instead evaluate food healthiness using "the more diverse (un)healthy ingredients in the food, the more (un)healthy the food" intuition. Our prediction is based on the fact that understanding nutritional value requires a certain level of knowledge to judge food healthiness. ${ }^{1,28}$ Not having a standard for judging the information makes it difficult to evaluate (ie, low in evaluability) ${ }^{29}$ such as evaluating whether the amount of saturated fat contained in a single serving pizza is appropriate. Thus, it is predicted that the person will use the number of unhealthy toppings (ie, three (sausage, bacon, and ham) vs one (sausage)) as a diagnostic cue rather than its saturated fat content (ie, $15 \mathrm{~g}$ ) when judging healthiness of the pizza. Consequently, belief in "the greater the number of (un)healthy ingredients, the more (un)healthy food" intuition acts as a bias resulting in the heuristic processing of food:

H1: Perceived (un)healthiness of food will be higher when the food contains multiple vs single (un)healthy ingredients.

\section{Moderating Role of Health Consciousness}

Health consciousness refers to the degree to which individuals attempt to take health-related actions. ${ }^{30}$ Healthconscious consumers are concerned with their health status and endeavor to improve their health by performing wellness behaviors and seeking health information. ${ }^{31-33}$ Thus, by its nature, health-conscious people are motivated to evaluate whether the food is beneficial for their health.

Our research proposes that health-conscious people are more likely to perceive food ingredients as salient information as compared to those with less health consciousness. Alba and Hutchinson ${ }^{34}$ suggested that the frequency of encountering certain information leads to an increased ability to categorize items specifically and perceiving differences among the items. Prior research in the food domain has also shown that interest in health leads to higher awareness on the distinctness of food ingredients. ${ }^{35}$ Thus, health-conscious people are more able to perceive the distinctness of each ingredient due to their greater familiarity with ingredients information. Furthermore, because the words distinctness and salience are interrelated, ${ }^{36}$ being aware of the distinctness of each ingredient leads to perceiving ingredients as salient information. For instance, Krüger, Mata and Ihmels ${ }^{37}$ examined that when evaluating a product, distinctness perception of an item induces such item to be perceived as a salient cue. Thus, it can be concluded that the enhanced ability to distinguish differences between ingredients in food products due to health consciousness renders the given information salient. 
Moreover, health-conscious individuals who actively exercise self-control are susceptible to health-related cues. ${ }^{38-40}$ Ironically, this tendency increases the likelihood of engaging in heuristic processing by using ineffective or inappropriate information. ${ }^{41}$ For example, compared to people with low health consciousness, those with high health consciousness are more likely to misjudge food products through invalid cues, such as food product name (eg, "candy" chews vs "fruit" chews), ${ }^{38}$ health claim (eg, "low-fat" nutrition labels), ${ }^{43}$ and numerosity of nutrition information (eg, 2200 kilocalories vs 9200 kilojoules). ${ }^{44}$

Building on these findings, this research argues that people with high health consciousness will show higher susceptibility to ingredients information than those with low health consciousness. Thus, our proposed "multiple ingredients effect" will be manifested strongly by consumers with high health consciousness. Based on the theoretical considerations above, we draw the following hypothesis:

$\mathrm{H} 2$ : The multiple ingredients effect will be greater for high (vs low) health-conscious people.

We have argued so far that when assessing food healthiness, information on food ingredients is more salient and distinguishable to highly health-conscious people as compared to less health-conscious people. Thus, it is predicted that the extent to which individuals perceive food ingredients as a salient cue engenders the difference between low and high health-conscious people.

It is possible to examine the moderating role of health consciousness level on the multiple ingredients effect by presenting the ingredients information as salient regardless of health consciousness level. Considering that the familiarity to information on certain items enables individuals to differentiate among the information, ${ }^{34}$ the classification of superordinate items could make each item appear to be salient and distinguishable from one another even for those less conscious of health. Thus, food healthiness perception of less health-conscious people will mirror that of more conscious people. As such, it is predicted that the salience of the superordinates at the learning phase leads to no difference in perceived healthiness regardless of dispositional health consciousness level. On the basis of the discussions above, the following hypothesis is proposed:

H3: The moderation effect of health consciousness will be weakened when increasing the salience of ingredients.

Previous research has argued that people process information heuristically with reliance on their lay beliefs when people lack evaluability to process information. ${ }^{4,9,10}$ Thus, the current research predicts that enhancing the evaluability of hard-to-evaluate nutrition information by AI leads to less reliance on "the greater the diversity of (un)healthy ingredients, the more (un)healthy food" intuition, rendering no multiple ingredients effect. Specifically, people would be less likely to be biased by the intuition when unfamiliar but core information (eg, fat content) is provided along with reference information (eg, recommended daily fat intake). In line with our theorizing, Viswanathan and Hastak $^{45}$ showed that presenting either the average amount of a nutrient or the range of nutrients for all brands of potato chips on the market as reference information improved consumers' understanding of the nutritional value of products. Thus, acquiring knowledge on the appropriate amount of daily fat intake would enable people to judge the perceived healthiness of foods without using a heuristic cue. This will lead to no multiple ingredients effect on the perceived healthiness of foods regardless of health consciousness level.

H4: The multiple ingredients effect will be attenuated when enhancing the evaluability of hard-to-evaluate nutrition information.

\section{Overview of Studies}

In the following section, two pretests and four experiments are reported in support of our hypotheses. Our research demonstrates the multiple ingredients effect that the number of ingredients (multiple vs single) acts as a cue for assessing food healthiness. Two pretests examine whether people have a lay belief about the association between the number of ingredients and food healthiness before testing our main hypotheses. Study 1 tests our primary effect of the number of ingredients (multiple vs single) on the perceived unhealthiness of vice food (H1) and the moderating role of health consciousness (H2). Study 2 replicates the multiple ingredients effect in the virtue food domain and rule out a subadditivity-based alternative account. Study 3 investigates the moderating role of health consciousness level on the multiple ingredients effect by directly increasing the salience of ingredients to both low and high health-conscious people (H3). Finally, unlike Studies 1-3 that have no reference information of unfamiliar nutritional value, Study 4 identifies the boundary condition to the multiple ingredients effect by enhancing the evaluability of unfamiliar nutrition information in the context of AI-based self-service (H4). The study procedures were in accordance with ethical standards. Studies from 
1-4 were approved by the Institutional Review Board of Korea University in South Korea. All participants were informed of the research process and provided written informed consent in accordance with the Declaration of Helsinki.

\section{Pretest: Lay Belief Testing}

To provide preliminary evidence for our main hypothesis that perception of food unhealthiness is greater when the same amount of saturated fat of ingredient is divided into multiple versus single ingredients, the current research conducts two pretests. These pretests examine whether people have a lay belief about the relationship between the number of (un) healthy ingredients and food healthiness.

\section{Method and Result}

A total of 101 individuals from Amazon Mechanical Turk $\left(54.5 \%\right.$ women; $\left.M_{\text {age }}=38.46\right)$ participated in this study. Participants were asked to indicate their opinion about the relationship between the number of unhealthy ingredients and food unhealthiness using one question ("the more the number of unhealthy ingredients (eg, bacon, ham, etc) in the food, the more unhealthy food it is"). The participants could respond with "agree," "disagree," or "not sure." The result showed that more people agreed $(80.2 \%)$ with the statement than disagreed $(10.9 \%)$ or indicated not sure $(8.9 \%)$ about the statement $\left(\chi^{2}(2)=99.88, \mathrm{p}<0.001\right)$. Separate test $(\mathrm{N}=$ $100,58 \%$ women, $\left.M_{\text {age }}=36.13\right)$ in the healthy food domain ("the more the number of healthy ingredients in the food, the more healthy food it is") also showed similar results; agreed $(87 \%)$, disagreed $(9 \%)$, not sure $(4 \%)\left(\chi^{2}(2)=\right.$ 129.98, $p<0.001)$. As predicted, the results indicate that a majority believe that there is a positive relationship between the number of (un)healthy ingredients and food (un)healthiness. Based on this initial support for our prediction, next studies test whether people use this lay belief in assessing food healthiness even though nutritional value (eg, total fat) is presented and this reliance on lay belief leads to biased food decision-making.

\section{Study I: Single versus Multiple Ingredients}

The purpose of Study 1 is to provide preliminary evidence that perception of food unhealthiness is greater when the same amount of saturated fat of ingredients is divided into multiple versus single ingredients. The study manipulated the number of unhealthy ingredients (three vs one), while the sum of saturated fat of ingredients was equal across conditions.

\section{Method}

A total of 311 participants $\left(40.2 \%\right.$ women; $\left.M_{\text {age }}=37.11\right)$ were recruited from Amazon MTurk. They were randomly assigned to one of the four between-subjects design conditions: one multiple ingredient and three single ingredient conditions. The multiple ingredients condition presents that the pizza consists of three meat toppings, whereas the single ingredient conditions present that the pizza consists of one out of three toppings used in the multiple ingredients condition.

Participants were presented with part of a newspaper article that explained the health risk of overconsuming processed meat. The article stated that processed meat contains high saturated fat and excessive consumption of processed meat increases the risk of obesity, diabetes, and some cancers. Below the article, a meat pizza was shown with nutrition information of saturated fat content (see Figure 1). Specifically, in the multiple ingredients condition, a saturated fat amount of sausage $(5 \mathrm{~g})$, ham $(5 \mathrm{~g})$ and bacon $(5 \mathrm{~g})$ was presented, respectively, below the picture of a meat pizza. However, one of each processed meat was presented in the single ingredient conditions. The saturated fat amount was presented as $15 \mathrm{~g}$ in the three single ingredient conditions.

Afterward, the perceived unhealthiness of pizza was measured on a 7-point Likert scale adapted from Lillis, Luoma, Levin, and Hayes ${ }^{46}$ ("Eating this pizza will make me fat."). Additionally, the study assessed the participants" levels of consciousness on maintaining good health on a 7-point Likert scale (2-item; $\alpha=0.94$ ) adapted from Tudoran, Olsen, and Dopico: ${ }^{47}$ "Maintaining health is very important to me," and "Health means a lot to me." Lastly, participants answered standard demographic questions.

\section{Results}

An one-way ANOVA showed a significant main effect of the number of ingredients on perceived unhealthiness $(F(3$, $\left.307)=7.90, p=0.023, \eta^{2}=0.03\right)$. Participants in the multiple ingredients condition $(M=5.60, S D=1.35)$ showed higher perception of being fat than those in the single ingredient conditions combined $(M=4.99, S D=$ $1.63 ; F(1,309)=8.80, p=0.003$, Cohen's $d=0.41)$, in support of H1. Comparisons across the four conditions showed that the perception of being fat in the multiple 


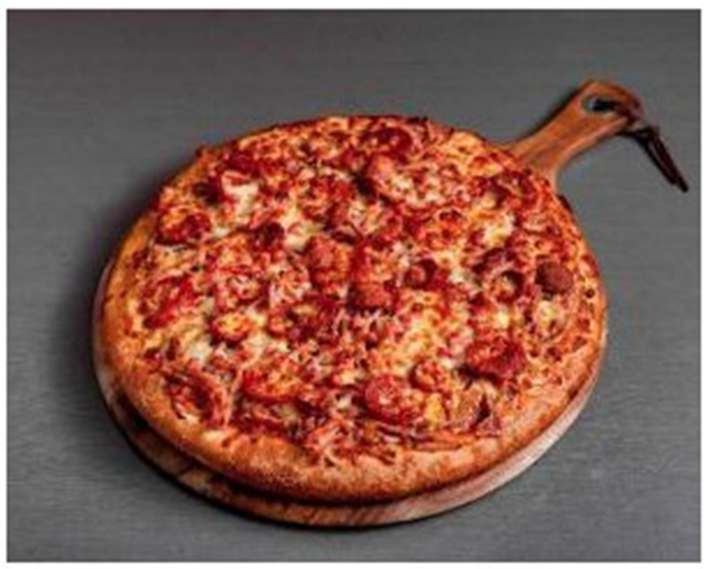

\section{The ultimate meat pizza \\ topped with sausage}

\section{- Saturated fat information (1 slice): sausage: $15 \mathrm{~g}$}

Figure I Single ingredient (sausage) condition in Study I.

ingredients condition was significantly higher than the sausage $(M=5.12, S D=1.60 ; F(1,307)=3.56, p=0.060, d=$ $0.32)$, ham $(M=4.96, S D=1.57 ; F(1,307)=6.37, p=$ $0.012, d=0.44)$, and bacon $(M=4.89, S D=1.72 ; F(1,307)$ $=8.19, p=0.005, d=0.46)$ conditions. The differences between the sausage $(M=5.12, S D=1.60)$ and ham $(M=$ $4.96, S D=1.57)$ conditions, sausage and bacon $(M=4.89$, $S D=1.72)$ conditions, and ham and bacon conditions were not significant $(F \mathrm{~s} \leq 0.85, p \mathrm{~s} \geq 0.358)$.

To test $\mathrm{H} 2$, a regression was conducted with the number of ingredients $(-1=$ multiple ingredients condition, +1 $=$ single ingredient condition), health consciousness (mean-centered), and the interaction as independent variables and perceived unhealthiness as dependent variable. The main effect of health consciousness was marginally significant $(\beta=0.16, S E=0.09, p=0.077)$. The main effect of the number of ingredients was significant $(\beta=$ $-.31, S E=0.10, p=0.003)$. Most importantly, the interaction between consciousness level and the number of ingredients was significant $(\beta=-.17, S E=0.09, p=$ 0.054).

We further analyzed the significant interaction effect by conducting a floodlight analysis using the Johnson-Neyman technique. ${ }^{48}$ The floodlight analysis proposed by Spiller et $\mathrm{al}^{49}$ allows us to identify the regions along with health consciousness where the simple effect of the multiple ingredients is significant and the range where it is not. The results revealed that the Johnson-Neyman point for significance $(p<0.05)$ for health consciousness moderator occurs when mean-centered health consciousness was greater than -.52 ( $t=-1.97, p=0.05$, see Figure 2). Specifically, healthconscious people had greater perception of unhealthiness when ingredients are presented with multiple (vs single) items. However, the number of ingredients did not influence perception of food unhealthiness for the low healthconscious people. These results supported $\mathrm{H} 2$.

\section{Discussion}

The results of Study 1 provide an initial demonstration of our proposed effect that people perceived higher food unhealthiness when the number of unhealthy ingredients was presented in multiple than when presented singly. Moreover, this study examined that the influence of the number of unhealthy ingredients on food healthiness perception was relatively greater for people who are highly 


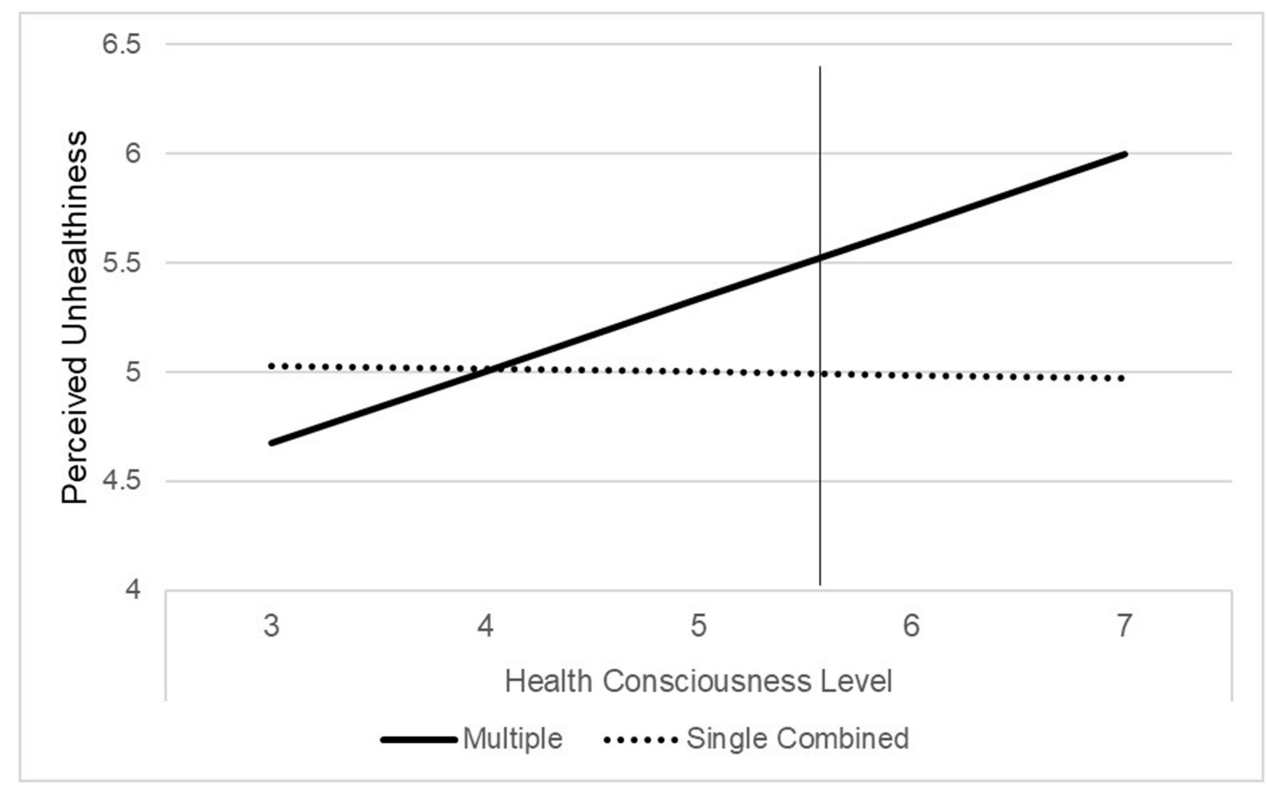

Figure 2 Floodlight analysis showing the interaction effect of health consciousness and the number of ingredients on perceived unhealthiness of pizza (Study I).

conscious of health compared to those who are less conscious of health.

\section{Study 2: Virtue Food Domain}

Study 2 has two main objectives. While Study 1 shows the impact of the number of unhealthy ingredients with vice food (ie, meat toppings on a pizza), the current study tests whether the multiple ingredients effect can be held in the virtue food domain. That is, it is predicted that the more diverse the healthy ingredients in the food, the greater perception of healthiness, especially for those who are highly conscious of their health. Additionally, one may argue that higher perceived healthiness of multiple ingredients in the previous study can be driven by the subadditivity effect of polyunsaturated fat content (eg, $f(5 \mathrm{~g})+f(5 \mathrm{~g})+f(5 \mathrm{~g})>f(15 \mathrm{~g}))$. This subadditivity effect is based on the notion that the total value is judged to be smaller than the sum of its parts. ${ }^{50}$ Hence, to rule out the subadditive-based alternative explanation, the study includes another multiple ingredients condition in which polyunsaturated fat content is indicated as a total. It is expected that perception of food healthiness in the multiple ingredients with total fat content $(15 \mathrm{~g})$ condition would not be different from the existing multiple ingredients with separate fat content $(5 g+5 g+5 g)$ condition, thereby indicating that the multiple ingredients effect is not driven by the subadditivity effect.

\section{Method}

A total of 274 participants $\left(48.2 \%\right.$ women; $\left.M_{\text {age }}=36.89\right)$ were recruited from Amazon MTurk. Participants were randomly assigned to one of the five between-subjects design conditions: two multiple ingredient and three single ingredient conditions. The only difference with Study 1 is that "multiple ingredients with the total fat" condition is included.

The procedures were similar to those of Study 1. First, participants were presented with part of a newspaper article that nuts are one of the best sources of polyunsaturated fat. The article also explained that eating nuts has various health benefits, such as improving heart health, cholesterol level, and losing weight. Below the article, the nut granola bar was shown with nutrition information of polyunsaturated fat content (see Figure 3). Specifically, in the three nuts with the separate fat content condition, the polyunsaturated fat amount of three nuts was presented separately [ie, almonds (5g), cashews (5g), and walnuts (5g)]. However, the total amount of polyunsaturated fat (15g) was presented in the three nuts with the total fat content condition [ie, almonds + cashews + walnuts $(15 \mathrm{~g})]$. The total amount of polyunsaturated fat $(15 \mathrm{~g})$ was presented in the three single-nut (almonds, cashews, walnuts) conditions.

Next, participants indicated perceived healthiness of the granola bar on a 7-point Likert scale adapted from Lillis et $\mathrm{al}^{46}$ (2-item; $\alpha=0.83$; "Eating this granola bar will be good for my health," and "I would be healthier if I eat this granola bar"; higher scores indicate greater perception of healthiness). Similar to Study 1, the participants assessed levels of consciousness in maintaining good health and 


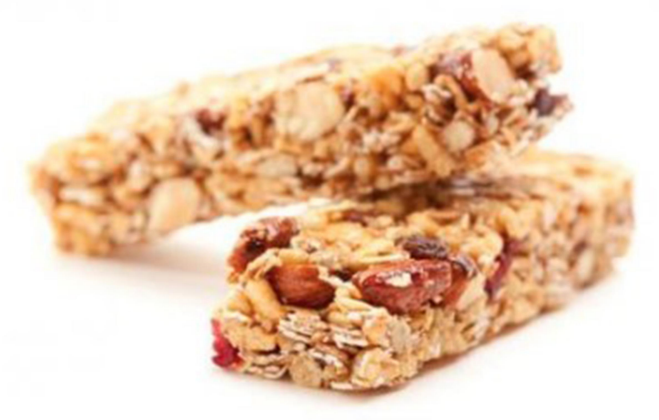

Combination of Almonds, Cashews, and Walnuts

- polyunsaturated fat contents (1 bar):

almonds: $5 \mathrm{~g}$,

cashews: $5 \mathrm{~g}$,

walnuts: $5 \mathrm{~g}$

Figure 3 Multiple ingredients (three nuts with separate fat content) condition (Study 2).

healthy diet on a 7-point Likert scale (2-item; $\alpha=0.94)$ adapted from Tudoran et al: ${ }^{47}$ "Maintaining health is very important to me," and "Healthy diet means a lot to me."

\section{Results}

Similar to the previous study, an one-way ANOVA showed that there is a significant main effect of the number of ingredients on perceived healthiness $(F(4,269)=4.50, p=$
$0.002, \eta^{2}=0.06$, see Figure 4). Consistent with H1, participants in the multiple ingredients with separate and combined fat content conditions $(M=5.35, S D=1.11)$ exhibited higher perception of being healthy than those in the single ingredient conditions combined $(M=4.76, S D=1.22 ; F(1,272)=$ $16.60, p<0.001, d=0.51)$. Importantly, no significant difference was observed between the multiple ingredients with separate $(M=5.34, S D=1.07)$ and total $(M=5.35$,

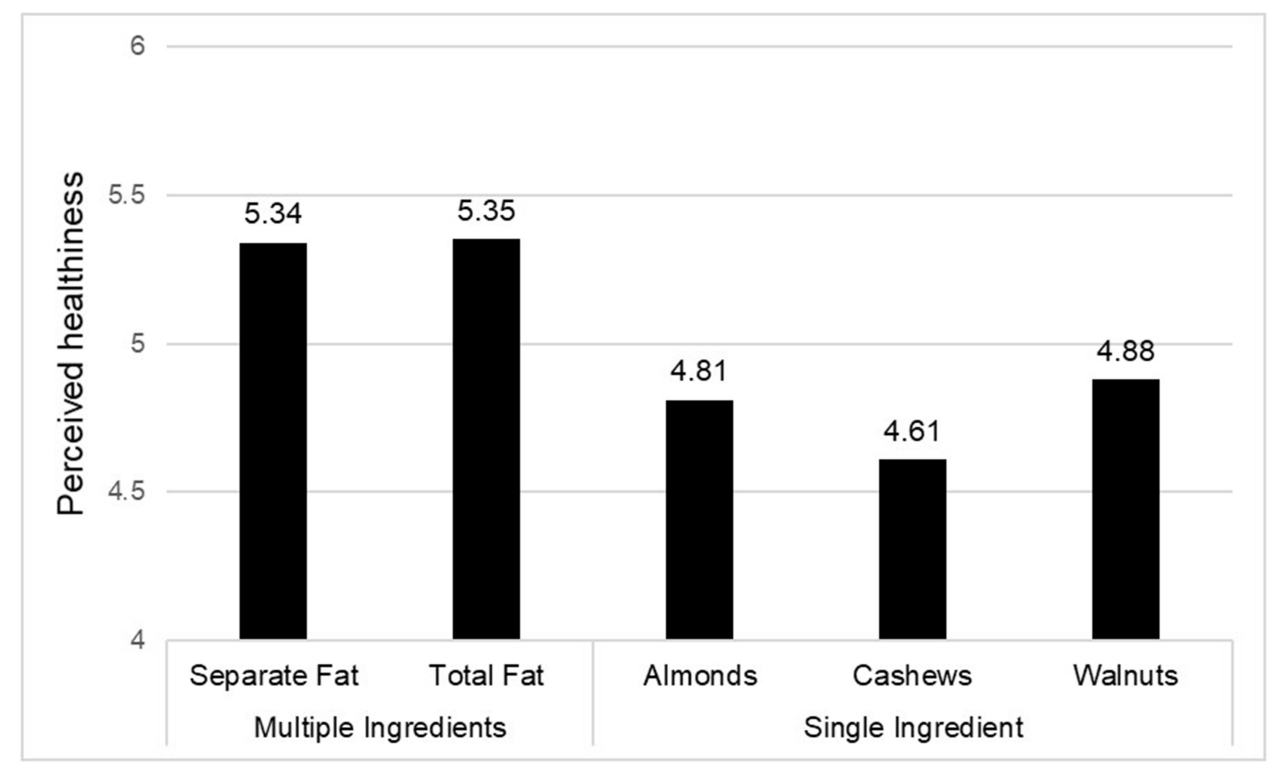

Figure 4 The impact of the number of ingredients on perceived healthiness (Study 2). 
$S D=1.16)$ fat content conditions $(F(1,269)=0.002, p=$ 0.964). Additionally, the differences between the almonds $(M=4.81, S D=1.10)$ and cashews $(M=4.61, S D=1.37)$ conditions, almonds and walnuts $(M=4.88, S D=1.17)$ conditions, and cashews and walnuts conditions were not significant $(F \mathrm{~s} \leq 1.36, p \mathrm{~s} \geq 0.245)$.

As in Study 1, a regression was performed with the number of ingredients $(-1=$ multiple ingredients conditions, $+1=$ single ingredient conditions), health consciousness (mean-centered), and the interaction as the independent variables and perceived healthiness as the dependent variable. The main effect of health consciousness was not significant $(\beta=0.06, S E=0.06, p=0.265)$. However, the main effect of the number of ingredients was significant $(\beta=-.29, S E=0.07, p<0.001)$. Most importantly, the interaction between consciousness level and the number of ingredients was significant $(\beta=-.11, S E=0.06$, $p=0.049$ ).

A floodlight analysis was conducted to examine whether the obtained multiple ingredients effect is moderated by the health consciousness level. Specifically, the results revealed that the Johnson-Neyman point for significance $(p<0.05)$ for health consciousness moderator occurs when mean-centered health consciousness was greater than -.94 $(t=-1.97, p=$ 0.05 , see Figure 5). Similar to the findings of Study 1 , the multiple ingredients effect was more pronounced in participants with relatively high (vs low) health consciousness, showing that they are more likely to perceive that eating granola bar makes them healthy when the number of healthy ingredients is multiple (vs single). These results confirmed $\mathrm{H} 2$.

\section{Discussion}

Study 2 replicated the results of Study 1 in the virtue food domain and confirmed the robustness of the multiple ingredients effect by showing that the healthiness perception of food was greater when the number of healthy ingredients was presented as multiple versus a single ingredient. In addition, this study finds that the multiple ingredients effect on perceived healthiness could not be attributed to the subadditivity of polyunsaturated fat content because no difference was observed in the healthiness perception between multiple ingredients presented with separate fat content and those presented with total fat content. The next study tests whether the extent to which perceiving ingredients information as salient causes different perception of food healthiness between low and high health-conscious people.

\section{Study 3: Increasing the Salience of Ingredients}

The objective of Study 3 is to investigate what drives the difference in food healthiness perception between high and low health-conscious people. Our findings that the health consciousness moderates the multiple ingredients effect arise from

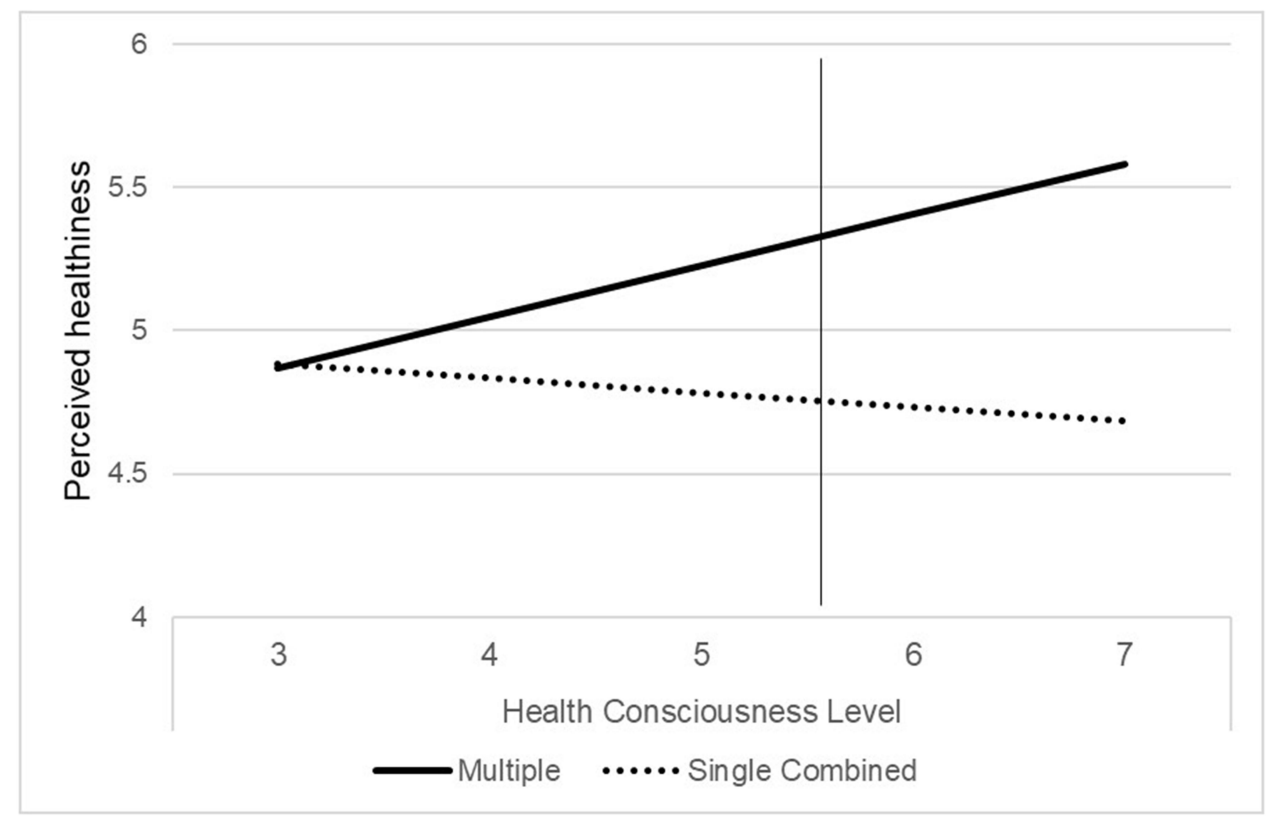

Figure 5 Floodlight analysis showing the interaction effect of health consciousness and the number of ingredients on perceived healthiness of granola bar (Study 2). 
the notion that high health-conscious people are more sensitive to ingredients information than low health-conscious people. This notion suggests that high health-conscious people are more likely to perceive ingredients as a more salient cue compared to those with low health consciousness. Thus, to test $\mathrm{H} 3$ that the degree to which ingredients salience moderates the multiple ingredients effect, ingredients information was manipulated into being perceived as more salient regardless of individual health consciousness level. Specifically, before viewing the ingredients information of pizza, the participants were presented with subordinates (eg, cooked sausage bologna, dry-cured ham - prosciutto, side bacon - pancetta) as stemming from each superordinate category (eg, sausage, ham, bacon). It is expected that the classification of superordinates (ie, sausage, ham, and bacon) makes each processed meat seem to be salient and distinguishable from each other even for people with less health consciousness.

\section{Method}

A total of 255 participants $\left(52.2 \%\right.$ women; $\left.M_{\text {age }}=39.97\right)$ were recruited from Amazon MTurk. As in Study 1, they were randomly assigned to one of the four between-subjects design conditions: one multiple ingredient and three single ingredient conditions. The procedures were similar to those of Study 1, with two exceptions for manipulation of ingredient salience. First, participants were presented with part of a newspaper article that showed several types of processed meats. The article explained that sausage, ham, and bacon are largely three types of processed meats that contain saturated fat. Below the article, specific classifications of sausage, ham, and bacon are presented with a diagram (see Figure 6). Second, to directly measure perceived healthiness, a new item asking whether eating the pizza is perceived as bad for health is added.

Similar to Study 1, the saturated fat amount $(5 \mathrm{~g})$ of each processed meat was presented separately in the multiple ingredients condition. In the three single ingredient conditions, the total amount of saturated fat $(15 \mathrm{~g})$ was presented. Next, participants indicated perceived unhealthiness of the pizza on a 7-point Likert scale (2-item; $\alpha=0.67$; "Eating this pizza will make me fat," and "Eating this pizza would be bad for my health"; higher scores indicate a greater perception of unhealthiness). Again, the study assessed the participants' levels of consciousness on maintaining good health ( $\alpha$ $=0.94$ ) as used in previous studies.

\section{Results}

An one-way ANOVA on perceived unhealthiness indicated a significant main effect of the number of ingredients $(F(3$, $\left.251)=2.90, p=0.036, \eta^{2}=0.03\right)$. Participants showed higher perception of being unhealthy when they were presented with multiple ingredients $(M=5.56, S D=1.13)$ than single ingredient conditions combined $(M=5.05, S D=1.28 ; F(1,253)=$ $8.08, p=0.005, d=0.42)$, in support of H1. Comparisons across the four conditions showed that unhealthiness perception in the multiple ingredients condition was significantly greater than the sausage $(M=5.13, S D=1.29 ; F(1,251)=$ 4.03, $p=0.046, d=0.35)$, ham $(M=5.07, S D=1.28 ; F(1$, $251)=4.73, p=0.031, d=0.41)$, and bacon $(M=4.95, S D=$ $1.28 ; F(1,251)=7.63, p=0.006, d=0.51)$ conditions. No differences were observed between the sausage $(M=5.13, S D$ $=1.29)$ and ham conditions $(M=5.07, S D=1.28)$, sausage and bacon $(M=4.95, S D=1.28)$ conditions, and ham and bacon conditions $(F \mathrm{~s} \leq 0.61, p \mathrm{~s} \geq 0.435)$.

As in previous studies, a regression was performed with the number of ingredients $(-1=$ multiple ingredients condition, $+1=$ single ingredient conditions), health consciousness (mean-centered), and the interaction as the independent variables and perceived unhealthiness as the dependent variable. The main effect of health consciousness was not significant $(\beta=0.10, S E=0.08, p=0.202$ ). However, the main effect of the number of ingredients was
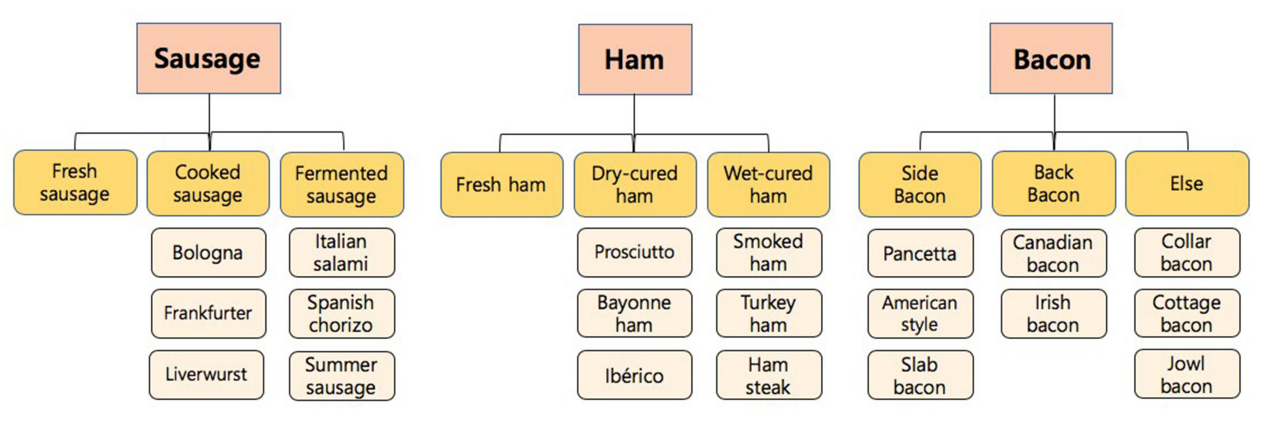

Figure 6 Classifications of sausage, ham, and bacon (Study 3). 
significant $(\beta=-.25, S E=0.09, p=0.005)$. Importantly, no significant interaction effect of the number of ingredients and perceived unhealthiness was observed $(\beta=0.002$, $S E=0.08, p=0.983$, see Figure 3). Thus, it can be concluded that the salience of each superordinate induces people to recognize ingredients (ie, sausage, ham, bacon) are distinct from each other regardless of their dispositional health consciousness level in support of H3.

\section{Discussion}

Findings from this study indicate that the difference in healthiness perception between high and low healthconscious people is driven by health-conscious people's higher awareness of differences among ingredients, which in turn leads them to perceive ingredients as more salient information. A post-test was further conducted to directly show that high health-conscious people are more likely to perceive unhealthy ingredients as distinct than low healthconscious people. As the word distinct refers to dissimilar, ${ }^{51}$ the extent of dissimilarity was measured as a proxy for measuring the perceived distinctness of ingredients. Thus, it is predicted that the higher the level of health consciousness, the greater perception of dissimilarity among the ingredients. A total of 108 participants $\left(40.7 \%\right.$ women; $\left.M_{\text {age }}=35.39\right)$ were recruited from Amazon MTurk. Five processed meats (ie, ham, bacon, sausage, corned beef, luncheon meat) are selected based on the classification of processed meat on the online newspaper article. ${ }^{52}$ Two out of five processed meats are paired together (eg, ham-bacon); therefore, a total of 10 combinations were presented in a random sequence. Participants indicated the perceived similarity of each pair based on their opinion on a 7-point scale $(1=$ dissimilar, $7=$ similar). Then, they rated health consciousness level on a 7-point Likert scale $(\alpha=0.81)$ as used in previous studies. Correlation analysis showed a significant negative correlation between average health consciousness and mean similarity perception of five processed meats $(r=-.19, p=$ 0.047). The results of the post-test complement Study 3 by showing that the greater the health consciousness people have, the more salience of each ingredient they perceive.

\section{Study 4: Increasing Evaluability of Fat with the Help of Artificial Intelligence}

The goal of Study 4 is to test $\mathrm{H} 4$ that the multipleingredients effect will be weakened when increasing the evaluability of hard-to-evaluate nutrition information. Saturated and polyunsaturated fat content of foods used in previous studies can be characterized as hard-to-evaluate information because prior knowledge on the appropriate daily fat intake is required when judging nutritional value. Thus, it is predicted that people are less likely to rely on "the greater the diversity of (un)healthy ingredients, the more (un)healthy food" intuition when they are able to evaluate unfamiliar nutrition information by AI (ie, chatbot-based kiosk), leading to no multiple ingredients effect. This study also tests whether the healthiness perception of foods has an impact on downstream consumer behavioral intention, which is the intention to purchase the food.

\section{Method}

A total of 363 participants ( $54.5 \%$ women; $M_{\text {age }}=39.79$ ) were recruited from Amazon MTurk. As in Study 1, they were randomly assigned to one of the four betweensubjects design conditions: one multiple ingredient and three single ingredient conditions.

Participants were asked to imagine that they went to a pizza restaurant and are about to order pizza using the self-ordering kiosk. After being shown the home screen of the kiosk, they were told that they had to decide on which pizza to choose. The screen shows the meat pizza with nutrition information including the main ingredients and their fat content (see Figure 7). Then, they were told that the restaurant provides recommended daily fat intake by a chatbot to enhance consumer welfare and well-being in addition to the saturated fat amount of each processed meat. Before checking out, the chatbot appears on the screen, showing "Tap me to see the recommended daily fat amount!" message. The participants assumed that they had to tap the chatbot to see the recommended daily fat amount. Then, the chatbot will provide the appropriate amount of daily fat intake by stating that the "Recommended daily fat intake (FDA) is on the next screen." "65g" was adopted based on FDA guidance for the appropriate daily fat intake. $^{53}$

After reading the scenario, participants indicated their perception of the unhealthiness of the pizza on a 7-point Likert scale as used in Study 3 (2-item; $\alpha=0.86$; higher scores indicate a greater perception of unhealthiness). Then, they rated intention to purchase the pizza on a 7-point Likert scale (2-item; $\alpha=0.98$; "How likely are you to purchase this meat pizza?" and "The likelihood of choosing this meat pizza is high."). They also reported consciousness level on maintaining good health $(\alpha=0.95)$ as used in prior studies. 
1. Meat Lovers' pizza

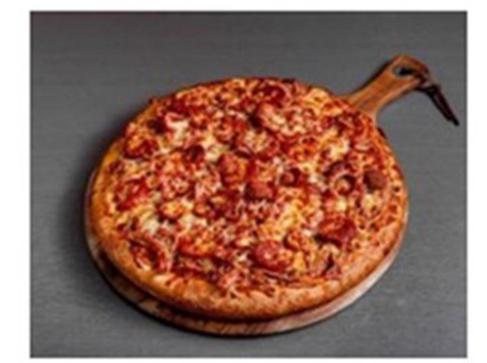

Cancel
- Topped with sausage, ham, bacon

- Saturated fat information (1 slice)

sausage: $\mathbf{5 g}$,

Ham: $\mathbf{5 g}$,

Bacon: $\mathbf{5 g}$

"Tap" me to see recommended daily fat amount!

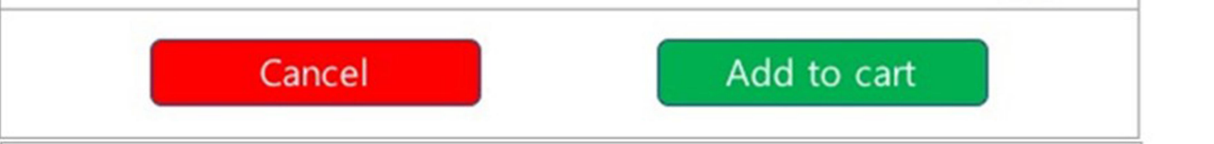

\section{Meat Lovers' pizza}

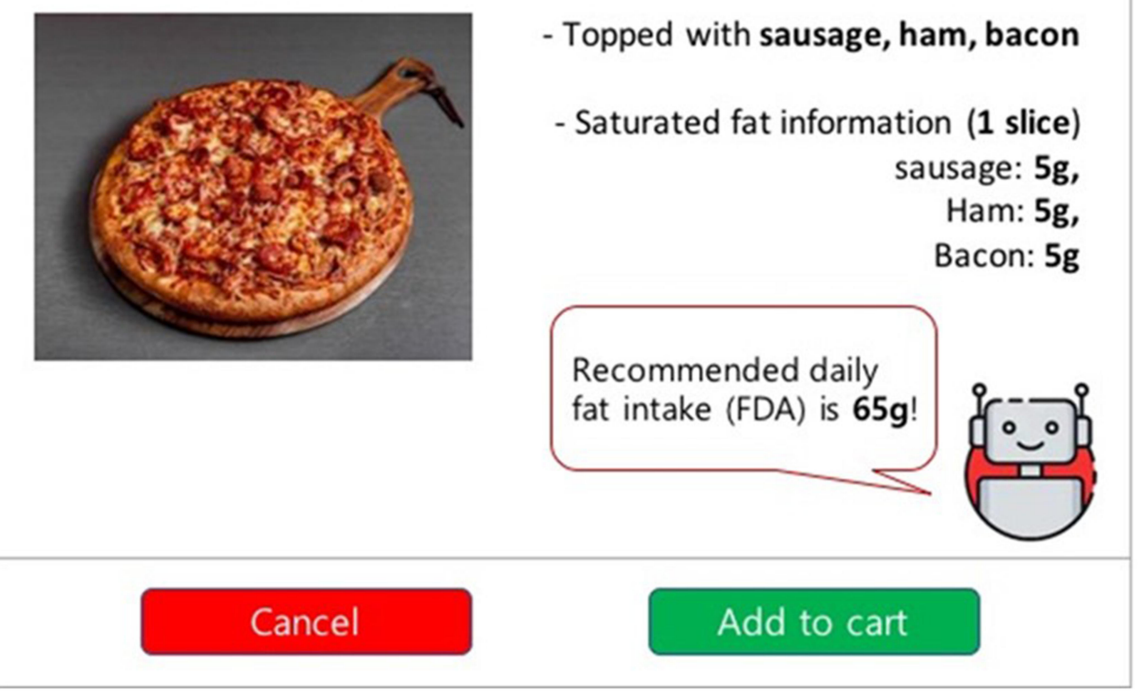

Figure 7 Multiple ingredients condition (Study 4).

\section{Results and Discussion}

Perceived Unhealthiness

An one-way ANOVA revealed that the main effect of the number of ingredients on perceived unhealthiness was not significant $(F(3,359)=0.39, p=0.763)$. There was no difference in perceived unhealthiness of foods was observed between the multiple ingredients condition $(M=4.48, S D=$ $1.50)$ and single ingredient conditions combined $(M=4.49, S D$ $=1.58 ; F(1,361)=0.002, p=0.969)$. Comparisons of four conditions revealed no significant differences in the perception of food unhealthiness $\left(M_{\text {multiple }}=4.48, S D=1.50 ; M_{\text {sausage }}=\right.$ $4.64, S D=1.51 ; M_{\text {ham }}=4.40, S D=1.72 ; M_{\text {bacon }}=4.44, S D=$ $1.51 ; F \mathrm{~s} \leq 1.05, p \mathrm{~s} \geq 0.305)$.
As in previous studies, a regression was conducted with the number of ingredients $(-1=$ multiple ingredients condition, $+1=$ single ingredient conditions), health consciousness (mean-centered), and the interaction as independent variables and perceived unhealthiness as dependent variable. The main effect of health consciousness was not significant $(\beta=0.13, S E=0.09$, $p=0.134)$. Also, the main effect of the number of ingredients was not significant $(\beta=0.01, S E=0.09$, $p=0.943)$. Most importantly, the interaction between the number of ingredients and health consciousness was not significant $(F(1,359)=0.42, p=0.520)$. 


\section{Purchase Intention}

A similar one-way ANOVA on purchase intention ratings showed that there was a no significant main effect of the number of ingredients $(F(3,359)=0.68, p=0.567)$. No significant difference in intention to purchase the food was observed between the multiple ingredients condition $(M=$ $4.28, S D=2.10)$ and single ingredient conditions combined $(M=4.41, S D=1.95)$. Also, there was no difference in purchase intention across the four conditions $\left(M_{\text {multiple }}=\right.$ $4.28, S D=2.10 ; M_{\text {sausage }}=4.20, S D=2.02 ; M_{\text {ham }}=4.44$, $\left.S D=1.79 ; M_{\text {bacon }}=4.59, S D=2.03 ; F \mathrm{~s} \leq 1.72, p \mathrm{~s} \geq 0.19\right)$.

A regression analysis was performed with purchase intention as the dependent variable. The results showed the main effect of the number of ingredients was not significant $(\beta=0.06, S E=0.12, p=0.641)$. However, a significant main effect of health consciousness $(\beta=-.27$, $S E=0.11, p=0.016)$ was observed, indicating that participants with high health consciousness were less likely to purchase unhealthy food than those with low consciousness. Also, as predicted, the interaction between the number of ingredients and health consciousness (meancentered) was not significant $(F(1,359)=0.84, p=$ 0.361 ). We confirmed the evaluability of nutrition information as a boundary condition for our multiple ingredients effect in the context of AI-based service agents. Thus, the findings of Study 4 provide evidence that the presence of reference information induces unbiased food decisionmaking.

\section{Discussion}

The current study examines the multiple ingredients effect, which posits that people make inferences about food (un) healthiness based on the diversity of (un)healthy ingredients. Across four experiments, our findings demonstrate that people rely on the lay belief that "the greater the number of (un)healthy ingredients, the more (un)healthy the food," although the number of ingredients is invalid information when judging food healthiness. Additionally, the multiple ingredients effect is moderated by the extent to which people are conscious of health-related issues. We initially examine that people indicate a higher perception of food unhealthiness when the number of unhealthy ingredients is presented as a multiple rather than single ingredient (Study 1). We replicate the multiple ingredients effect in the healthy food domain and eliminate an alternative explanation based on the subadditivity effect (Study 2). We also find that the difference in food healthiness perception between people who have high and low health consciousness is driven by the salience of each ingredient (Study 3). Finally, we identify the evaluability of the nutritional value as a boundary condition for our obtained effects in an AI-based self-service context (Study 4).

\section{Theoretical Contributions}

Our research contributes to previous literature by examining the relationship between heuristic processing and food decision-making. We propose and investigate the multiple ingredients effect that the perceived healthiness of foods differs depending on the sheer number of ingredients. Although a considerable number of studies have examined the effects of lay theories on consumers' food choice decision, ${ }^{4-7}$ this research suggests the number of ingredients as a novel cue that influences perception of food healthiness. Building on the findings of previous literature that presenting healthy ingredients on food health claims has a positive effect on consumers' perception of food, ${ }^{18,54}$ we confirmed and generalized the multiple ingredients effect across opposite food domains (ie, vice vs virtue food) and different types of food judgment tasks. In addition, although previous studies suggest that controlling lay intuitions may be beyond people's conscious awareness, ${ }^{6}$ our findings (Study 4) show a simple and actionable strategy to reduce reliance on their existing lay beliefs. Presenting reference information (eg, appropriate daily sodium intake) on unfamiliar nutrition information (eg, amount of sodium) of foods can lessen the multiple ingredients effect.

This research also contributes to the literature by examining the relationship between health consciousness and information processing. A great deal of controversy has been raised regarding whether health-conscious people engage in heuristic, ${ }^{41,42,55}$ or deliberative processing, ${ }^{12,56,57}$ when judging food. One stream of research suggested that as long as a useful clue that can serve as a basis for judgment exists, restrained (ie, health-conscious) people are less likely to be biased and thus make a deliberate and conscious judgment. ${ }^{57,58}$ This stream of research suggests that health-conscious people would be less likely to engage in heuristic processing when the presented information is relatively easy to interpret. ${ }^{44}$ However, another stream of research showed that highly health-conscious people tend to process information heuristically, relying on invalid cues, such as the mere name of the food product, health claim, and 
numerosity of food energy unit. ${ }^{41,43,44}$ The likelihood of engaging in heuristic processing increases especially when the objective meaning of the information (eg, conversion rate) is relatively difficult to understand.$^{44}$ One may argue that health consciousness and evaluability of health-related information are positively correlated. However, we note that the level of knowledge even varies among people who regard health as an important issue,${ }^{59}$ indicating that even health-conscious individuals are not able to process hard-to-understand information without specific criteria for judgment. In summary, our research contributes to the previous literature by suggesting that the degree to which highly health-conscious people engage in heuristic information processing differs depending on the evaluability of nutrition information.

\section{Managerial Implications}

The current findings have implications for marketers and policymakers to nudge consumers into making healthier food choices. Grocery store managers and advertising managers trying to promote healthy food choices would do better when implementing the "multiple ingredients effect" strategy. In particular, private brands of organic grocery stores (eg, Whole Food Market, Trader Joe's) tend to make health claims presenting particular ingredients on packages to emphasize the healthiness of their food products. Thus, highlighting the number of healthy ingredients (eg, rye flour, whole wheat flour, oats) in virtue foods (eg, multigrain bread) would have a considerable effect in the choice of healthy products rather than emphasizing their unfamiliar nutritional value (eg, dietary fiber: $5 \mathrm{~g}$ ).

A public health campaign can also improve consumer welfare by representing unhealthy food items as salient and distinguishable in the campaign message. For example, informing how processed meats are classified with levels of subordinates (eg, sausage - cooked sausage - bologna, ham dry-cured ham - prosciutto, bacon - side bacon - pancetta) in the message increases the salience of each item, which can exert the multiple ingredients effect on subsequent food choice decisions. Consequently, this strategic message can induce consumers to perceive vice foods (eg, meat pizza) made with unhealthy ingredients (eg, processed meats) as unhealthy regardless of their dispositional health consciousness.

Additionally, our research provides practical guidelines to both marketers and consumers in the domain of AI-based selfservice. With the rapid increase in the deployment of AIperformed service systems, marketers in the food industry can provide personalized nutrition information to consumers based on their order history. For example, if certain consumers repeatedly choose the pizza menu with the least number of meat toppings, the consumer can receive personalized reference information (eg, the average level of fat in similar products) with the help of AI-based service system. Marketers can help new consumers to make sound food choices by preventing reliance on eye-catching cues (eg ingredients) not only providing personalized reference information to particular consumers, but also by presenting reference information on hard-to-evaluate information via chatbots.

\section{Limitations and Future Research Directions}

Finally, our findings highlight directions for future research. In this research, we examine the boundary condition for the multiple ingredients effect by presenting reference information (ie, recommended daily fat intake) to enhance the evaluability of unfamiliar information. As previous research has shown that providing either the average amount of nutrient or the range of the nutrient of other brands affects the perception of food, ${ }^{45}$ other information that can be served as a reference may also enhance the evaluability of nutrition information. Thus, it is possible to examine other boundary conditions of our obtained effect as long as it can improve the evaluability of unfamiliar nutritional value. In addition, considering that the degree of ingredient multiplicity was manipulated by comparing three ingredients with one ingredient in all experiments, it might be interesting to examine the multiple ingredients effect with changes in the number of food ingredients (eg, one vs four vs seven vs ten). It is predicted that people would show diminishing sensitivity (ie, concave utility function) ${ }^{60}$ to the perceived healthiness of foods as the number of ingredients increased. Thus, it is worth examining the multiple ingredients effect with various sizes of the number to explore potential boundary conditions.

Moreover, while this research provides theorization on how the number of (un)healthy ingredients acts as means of assessing food healthiness, we did not directly test the source of the intuitive association between the number of healthy ingredients and food healthiness. Drawing from Haws et al's findings ${ }^{4}$ that people base the association between food healthiness and price on their prior exposures to restaurants and grocery stores and information from various media, we similarly predict that people gradually establish a link between the number of (un)healthy ingredients and food healthiness influenced by past experiences in grocery stores and 
information from food advertisements. This prediction is in line with previous research that the ease of retrieving certain information is highly affected by indirect experiences, such as media coverage. ${ }^{61}$ Although the impacts of lay beliefs on decision-making may occur unconsciously because such beliefs are established in cognitive structure over a long period, ${ }^{6}$ future research could explore the primary root of our multiple ingredients effect. In addition, we utilize the real food ingredient in order to ensure the ecological validity. Thus, it is worthwhile to replicate our multiple ingredients effect in the future research using fictitious ingredients (eg, ingredient $\mathrm{A}, \mathrm{B}$, and $\mathrm{C}$ ) to prevent other confounding factors.

\section{Acknowledgments}

This research has been financially supported by the Fellowship Program of the Center for Social value Enhancement Studies.

\section{Disclosure}

The authors report no conflicts of interest in this work.

\section{References}

1. Kim SY, Nayga RM, Capps O. The effect of food label use on nutrient intakes: an endogenous switching regression analysis. $J \mathrm{Agr}$ Resour Econ. 2000;25(1):215-231.

2. Rebollar R, Lidón I, Gil I, Martín J, Fernández MJ, Riveres CE. The influence the serving suggestion displayed on soft cheese packaging has on consumer expectations and willingness to buy. Food Qual Prefer. 2016;52:188-194. doi:10.1016/j.foodqual.2016.04.015

3. Variyam JN. Do nutrition labels improve dietary outcomes? Health Econ. 2008;17(6):695-708. doi:10.1002/hec.1287

4. Haws KL, Reczek RW, Sample KL. Healthy diets make empty wallets: the healthy $=$ expensive intuition. $J$ Consum Res. 2017;43 (6):992-1007.

5. Madzharov AV, Block LG. Effects of product unit image on consumption of snack foods. J Consum Psychol. 2010;20(4):398-409. doi:10.1016/j.jcps.2010.06.007

6. Raghunathan R, Naylor RW, Hoyer WD. The unhealthy = tasty intuition and its effects on taste inferences, enjoyment, and choice of food products. $J$ Mark. 2006;70(4):170-184. doi:10.1509/jmkg. 70.4 .170

7. Suher J, Raghunathan R, Hoyer WD. Eating healthy or feeling empty? How the "healthy = less filling" intuition influences satiety. $J$ Assoc Consum Res. 2016;1(1):26-40. doi:10.1086/684393

8. Food Navigator. Ingredients lists are powerful purchasing motivators: study finds consumer care more about ingredients than brand; 2018. Available from: https://www.foodnavigator.com/Article/2018/09/28/ Consumers-care-more-about-ingredients-than-brand-Survey. Accessed April 3, 2019.

9. Chaiken S. Heuristic versus systematic information processing and the use of source versus message cues in persuasion. J Pers Soc Psychol. 1980;39(5):752-766. doi:10.1037/0022-3514.39.5.752

10. Chen S, Chaiken S. The Heuristic-systematic model in its broader context. In: Chaiken S, Trope Y, editors. Dual-Process Theories in Social Psychology. New York: Guilford; 1999:73-96.
11. Haws G, Gámbaro A. Influence of gender, age and motives underlying food choice on perceived healthiness and willingness to try functional foods. Appetite. 2007;49(1):148-158. doi:10.1016/j.appet. 2007.01.006

12. Chandon P, Wansink B. The biasing health halos of fast-food restaurant health claims: lower calorie estimates and higher side-dish consumption intentions. J Consum Res. 2007;34(3):301-314. doi:10.10 86/519499

13. Provencher V, Polivy J, Herman CP. Perceived healthiness of food. If it's healthy, you can eat more! Appetite. 2009;52(2):340-344. doi:10.1016/j.appet.2008.11.005

14. Verain MC, Sijtsema SJ, Antonides G. Consumer segmentation based on food-category attribute importance: the relation with healthiness and sustainability perceptions. Food Qual Prefer. 2016;48(Pt A):99-106. doi:10.1016/j.foodqual.2015.08.012

15. Lee SM, Lee D. "Untact": a new customer service strategy in the digital age. Serv Bus. 2019;14:1-22. doi:10.1007/s11628-019-00408-2

16. Chandon P, Wansink B. Is obesity caused by calorie underestimation? A psychophysical model of meal size estimation. $J$ Mark Res. 2007;44(1):84-99. doi:10.1509/jmkr.44.1.84

17. Kozup JC, Elizabeth HC, Scot B. Making healthful food choices: the influence of health claims and nutrition information on consumers' evaluations of packaged food products and restaurant menu items. J Mark. 2003;67(2):19-34. doi:10.1509/jmkg.67.2.19.18608

18. Sütterlin B, Siegrist M. Simply adding the word "fruit" makes sugar healthier: the misleading effect of symbolic information on the perceived healthiness of food. Appetite. 2015;95:252-261. doi:10.1016/j. appet.2015.07.011

19. Petty RE, Cacioppo JT. The effects of involvement on responses to argument quantity and quality: central and peripheral routes to persuasion. J Pers Socl Psychol. 1984;46(1):69-81. doi:10.1037/ 0022-3514.46.1.69

20. Homer PM, Mukherjee S. The impact of dietary supplement form and dosage on perceived efficacy. J Consum Mark. 2018;34(6): 5063-5078.

21. Berger J, Draganska M, Simonson I. The influence of product variety on brand perception and choice. Mark Sci. 2007;26(4):460-472. doi: $10.1287 / \mathrm{mksc} .1060 .0253$

22. Oppewal H, Koelemeijer K. More choice is better: effects of assortment size and composition on assortment evaluation. Int J Res Mark. 2005;22(1):45-60. doi:10.1016/j.ijresmar.2004.03.002

23. Kahneman D. Attention and Effort. NJ: Prentice-Hall; 1973.

24. Kahn BE, Wansink B. The influence of assortment structure on perceived variety and consumption quantities. $J$ Consum Res. 2004;30(4):519-533. doi:10.1086/380286

25. Wansink B, Chandon P. Slim by design: redirecting the accidental drivers of mindless overeating. J Consum Psychol. 2014;24 (3):413-431.

26. Furnham A. Lay Theories: Everyday Understanding of Problems in the Social Sciences. Pergamon Press; 1988.

27. Tversky A, Kahneman D. Availability: a heuristic for judging frequency and probability. Cogn Psychol. 1973;5(2):207-232. doi:10.1016/0010-0285(73)90033-9

28. Drichoutis AC, Lazaridis P, Nayga RM. Nutrition knowledge and consumer use of nutritional food labels. Eur Rev Agric Econ. 2005;32 (1):93-118. doi:10.1093/erae/jbi003

29. Hsee CK. Attribute evaluability and its implications for joint-separate evaluation reversals and beyond. In: Kahneman D, Tversky A, editors. Choices, Values, and Frames. New York: Cambridge University Press; 2000:543-563.

30. Becker MH, Maiman LA, Kirscht JP, et al. The health belief model and prediction of dietary compliance: a field experiment. $J$ Health Soc Behav. 1977;18(4):348-366. doi:10.2307/2955344

31. Gould SJ. Consumer attitudes toward health and health care: a differential perspective. J Consum Aff. 1988;22(1):96-118. doi:10.1111/j.1745-6606.1988.tb00215.x 
32. Hu CW A new measure for health consciousness: development of a health consciousness conceptual model. Unpublished paper presented at: National Communication Association 99th Annual Convention; November; 2013; Washington, DC.

33. Kraft FB, Goodell PW. Identifying the health conscious consumer. Mark Health Serv. 1993;13(3):18-25.

34. Alba JW, Hutchinson JW. Dimensions of consumer expertise. J Consum Res. 1987;13(4):411-454. doi:10.1086/209080

35. Bornkessel S, Bröring S, Omta SO, et al. What determines ingredient awareness of consumers? A study on ten functional food ingredients. Food Qual Prefer. 2014;32:330-339. doi:10.1016/j.foodqual.2013. 09.007

36. Drager K, Kirtley MJ. Awareness, salience, and stereotypes in exemplar-based models of speech production and perception. In: Babel A, editor. Awareness and Control in Sociolinguistic Research. Cambridge: Cambridge University Press; 2016:1-24.

37. Krüger T, Mata A, Ihmels M. The presenter's paradox revisited: an evaluation mode account. J Consum Res. 2014;41(4):1127-1136. doi:10.1086/678393

38. Carels RA, Konrad K, Harper J. Individual differences in food perceptions and calorie estimation: an examination of dieting status, weight, and gender. Appetite. 2007;49(2):450-458. doi:10.1016/j. appet.2007.02.009

39. Fedoroff ID, Polivy J, Herman CP. The effect of pre-exposure to food cues on the eating behavior of restrained and unrestrained eaters. Appetite. 1997;28(1):33-47. doi:10.1006/appe.1996.0057

40. Fedoroff I, Polivy J, Herman CP. The specificity of restrained versus unrestrained eaters' responses to food cues: general desire to eat, or craving for the cued food? Appetite. 2003;41(1):7-13. doi:10.1016/ S0195-6663(03)00026-6

41. Coelho Do Vale R, Pieters R, Zeelenberg M. Flying under the radar: perverse package size effects on consumption self-regulation. J Consum Res. 2008;35(3):380-390. doi:10.1086/589564

42. Irmak C, Vallen B, Robinson SR. The impact of product name on dieters' and nondieters' food evaluations and consumption. J Consum Res. 2011;38(2):390-405. doi:10.1086/660044

43. Wansink B, Chandon P. Can "low-fat" nutrition labels lead to obesity? J Mark Res. 2006;43(4):605-617. doi:10.1509/jmkr.43.4.605

44. Wilcox K, Prokopec S, Morwitz VG, Kirmani A, Bagchi R. Restraint that blinds: attention narrowing and consumers' response to numerosity in self-control decisions. J Consum Res. 2018;46(2):371-387. doi:10.1093/jcr/ucy078

45. Viswanathan M, Hastak M. The role of summary information in facilitating consumers' comprehension of nutrition information. J Public Policy Mark. 2002;21(2):305-318. doi:10.1509/jppm.21.2. 305.17596

46. Lillis J, Luoma JB, Levin ME, et al. Measuring weight self-stigma: the weight self-stigma questionnaire. Obesity. 2010;18(5):971-976. doi:10.1038/oby.2009.353
47. Tudoran A, Olsen SO, Dopico DC. The effect of health benefit information on consumers health value, attitudes and intentions. Appetite. 2009;52(3):568-579. doi:10.1016/j.appet.2009.01.009

48. Johnson PO, Neyman J. Tests of certain linear hypotheses and their application to some educational problems. Stat Res Mem. 1936;1:57-93.

49. Spiller SA, Fitzsimons GJ, Lynch JG, McClelland GH. Spotlights, floodlights, and the magic number zero: simple effects tests in moderated regression. J Mark Res. 2013;50(2):277-288. doi:10.1509/ jmr. 12.0420

50. Tversky A, Koehler DJ. Support theory: a nonextensional representation of subjective probability. Psychol Rev. 1994;101(4):547-567. doi:10.1037/0033-295X.101.4.547

51. Dictionary.com. Distinct; 2012. Dictionary.com. Available from https://www.dictionary.com/browse/distinct. Accessed November 26, 2020.

52. Healthline. Why processed meat is bad for you; 2017. Available from: https://www.healthline.com/nutrition/why-processed-meat-isbad. Accessed December 5, 2019.

53. U.S. Food and Drug Administration/Center for Food Safety and Applied Nutrition. A food labeling guide; 2013. Available from: https://www.fda.gov/media/81606/download. Accessed January 9, 2020.

54. Paul GL, Ink SL, Geiger CJ. The Quaker oats health claim: a case study. J Nutraceuticals Funct Med Foods. 1999;1(4):5-32. doi:10. 1300/J133v01n04 02

55. Scott ML, Nowlis SM, Mandel N, et al. The effects of reduced food size and package size on the consumption behavior of restrained and unrestrained eaters. J Consum Res. 2008;35(3):391-405. doi:10.1086/ 591103

56. Cornil Y, Ordabayeva N, Kaiser U, et al. The acuity of vice: attitude ambivalence improves visual sensitivity to increasing portion sizes. J Consum Psychol. 2014;24(2):177-187. doi:10.1016/j.jcps.2013. 09.007

57. Hofmann W, Friese M, Strack F. Impulse and self-control from a dual-systems perspective. Perspect Psychol Sci. 2009;4(2):162-176.

58. Salmon SJ, Fennis BM, de Ridder DT, et al. Health on impulse: when low self-control promotes healthy food choices. Health Psychol. 2014;33(2):103-109. doi:10.1037/a0031785

59. Walters A, Long M. The effect of food label cues on perceptions of quality and purchase intentions among high-involvement consumers with varying levels of nutrition knowledge. J Nutr Educ Behav. 2012;44(4):350-354.

60. Tversky A, Kahneman D. Prospect theory: an analysis of decision under risk. Econometrica. 1979;47(2):263-291. doi:10.2307/1914185

61. Combs B, Slovic P. Newspaper coverage of causes of death. Journal Q. 1979;56(4):837-849.
Psychology Research and Behavior Management

\section{Publish your work in this journal}

Psychology Research and Behavior Management is an international, peer-reviewed, open access journal focusing on the science of psychology and its application in behavior management to develop improved outcomes in the clinical, educational, sports and business arenas. Specific topics covered in the journal include: Neuroscience, memory and decision making; Behavior modification and management; Clinical applications; Business and sports performance management; Social and developmental studies; Animal studies. The manuscript management system is completely online and includes a very quick and fair peer-review system, which is all easy to use. Visit http://www. dovepress.com/testimonials.php to read real quotes from published authors. 